\title{
Delay Analysis Methods for Construction Projects: Mathematical Modelling
}

\author{
Alena Vasilyeva-Lyulina ${ }^{1}$, Masamitsu Onishi ${ }^{2}$ and Kiyoshi Kobayashi ${ }^{3}$ \\ ${ }^{I}$ Project Advisors International LLC \\ ${ }^{2,3}$ Kyoto University \\ 1alenkavl@hotmail.com, 20nishi.masamitsu.7e@kyoto-u.ac.jp, \\ ${ }^{3}$ kobayashi.kiyoshi.6n@kyoto-u.ac.jp
}

\begin{abstract}
Delay is quite common in construction project. A variety of factors contribute to the delay of project completion in the complex interdependencies of a number of tasks. Hence determining the contractual responsibility of delay is the most likely source of dispute in construction projects. Methods used for forensic investigation of delay are called as 'delay analysis methods'. Although authoritative societies such as SCL (Society of Construction Law) and AACEI (Association for the Advancement of Cost Engineering International) have published guidance for implementing delay analysis methods, those are explained by a natural language, i.e. English, not by a formal language. On the other hand, process control of construction projects heavily relies on CPM programming based on the network modelling technique of which concept is described in a formal manner. The ambiguity of methods due to the lack of guidance on delay analysis methods described in a formal language may allow delay analysts to implement in different ways. Utilizing the mathematics as a formal language to explain delay analysis methods should contribute to reduce the degree of ambiguity of delay analysis methods and hence the possibility of dispute occurrence. This paper aims to formalising delay analysis methods commonly applied in practice employing the mathematical language.
\end{abstract}

Keywords: construction project, contrapct management, delay analysis, mathematical formulation

\section{Introduction}

Delay is quite common in construction projects. Delay can be caused by the Employer, the Contractor, and the third party or the force majeure that the parties to a contract cannot control. Delay to completion of a project could bring significant losses on society as well as the project cost. The damage caused by a project delay is compensated by the Contractor in principle in the form of 'liquidated damage'. However, the Contractor shall claim the extension of time or the recovery of financial burden if the Employer is liable for the cause of delay. In fact, a variety of factors contribute to the delay of project completion in complex interdependencies of a number of tasks. Hence determining the contractual responsibility of delay is the most likely source of dispute in construction projects for transportation infrastructure. Methods used for forensic investigation of delay are called as 'delay analysis methods'.

Delay analysis methods are applied to prepare the logical basis to persuade their claims concerning the extension of time and financial burden. More precisely, delay analysis refers to a forensic investigation into the issue of what has caused a project to run late, for the purpose of 1) establishing lines of investigation, 2) demonstrating entitlement and 3) presenting the case one is seeking to prove 0 . Delay analysis methods must provide logic of 'cause and effect' relationship, identifying what events contributed to delay and what is the effect of each event. 
Various delay analysis methods have been innovated and each method has its pros and cons for the practical application. Therefore, delay analysis methods are not such analytical instruments that only the best one is universally applied. Despite of the variety of delay analysis methods, there has been little guidance until recently, as to how each method should be carried out. Difference of methods is recognised by names such as 'impacted as-planned', 'time impact analysis' and 'collapsed as-built'. These names just provide a general indication of the approach being adopted by the delay analyst [1]. Authoritative societies such as SCL (Society of Construction Law) and AACEI (Association for the Advancement of Cost Engineering International) have published the guidance [2][3], it is explained by a natural language, i.e., English, not by a formal language.

On the other hand, process control of construction projects heavily relies on CPM (Critical Path Method) programming. CPM is a system model to calculate the theoretical estimate of project period by inputting relevant variables concerning the project schedule and identifying the sequence of activities on which delay critically causes the delay to project completion. Therefore, the actual work of delay analysis is systematic based on the hard logic of CPM programming. Despite of the systematic nature of delay analysis, there has been no guidance on the implementation of delay analysis methods explained in a formal language such as a mathematical modelling. Explanation of delay analysis methods by the natural language cannot avoid the ambiguity when actually applied. The ambiguity of methods due to the lack of guidance explained in a formal language may allow delay analysts to implement in different ways even if they apply a method with the same 'name'. Utilizing the mathematics as a formal language to explain delay analysis methods should contribute to reduce the degree of ambiguity of delay analysis methods and hence the possibility of dispute occurrence. This paper aims to formalising delay analysis methods commonly applied in practice employing a formal language, i.e., formalising mathematical models.

The paper is organized as follows. 2. summarises the basic components of delay analysis methods including CPM programming, as-planed and as-built programme, delay events and types of delay analysis methods. 3. is the core part of the study which describe the analytical procedures of delay analysis methods commonly used in practice by mathematical representation. 4. concludes the paper by referring to the significance of our work in terms of the contribution to the delay analysis practice.

\section{Basic Components of Delay Analysis}

\subsection{CPM Programme}

Critical interests in a construction project include the estimate of necessary time for completing it, and identification of the sequence of activities on which delay causes the delay to project completion. A sound estimate of project time requires valid assumptions how the project is carried out. CPM (Critical Path Method) is a most commonly used modelling technique to calculate a project's critical path, activity event times, float time and hence the estimate time of the project. In addition, it must prospectively forecast the likely effect of changed circumstances. CPM sees a project as a composition of activities. CPM programming uses the network analysis technique based on primarily two variables: activity durations and activity relationships [1]. Given the set of activities involved in a project with those durations and activity relationships, the CPM uniquely derives the necessary time for completing the project. CPM provides a formal structure systematically describe the project schedule in a way that can simulate the time impact of any change in project assumptions. 
Formally, a CPM programme is any $\Gamma$ of the form

$$
\Gamma=\left(A,\left(d_{i}\right)_{i \in A}, \boldsymbol{P}\right),
$$

where $A$ is the set of all activities involved in a project and $d_{i}$ is the duration of activity $i$. $\boldsymbol{P}$ represents activity relationships. The logic of activity relationships is classified into four forms [4]:

- finish-to-start - Task B cannot start until Task A has finished

- start-to-start - Task B cannot start until Task A has started

- finish-to-finish - Task B cannot finish until Task A has finished.

- Start-to-finish - e.g., Task B cannot finish until Task A has started

For any CPM programme $\Gamma$, the necessary time for completing the project is uniquely calculated which is denoted by $\tau$. CPM is a method to formally describe any project scheduling no matter whether the schedule is just a plan or a fact.

\subsection{As-planned programme}

The as-planned programme is supposed to show the Contractor's true intentions, what activities were planned to be performed, when, in which sequence, etc. as detailed as possible. Usually it is made using available computer software based on a CPM programming. The date of project completion agreed on the contract is calculated based on the as-planned programme. It is not rare that the analyst may need to correct the baseline programme before relying on it in any substantive way due to inherent flaws or errors in a contractor's assumption [1]. If the completion date is determined based on the flawed as-planned program, the project time could be too short which results in delay to completion. Delay due to a flawed as-planned program does not entitle the Contractor to claim extension of time. Thus preparation of reliable as-planned programme is essential.

The as-planned programme is itself a theoretical model how a particular contractor would like to build a project. To build a valid as-planned programme, the contractor must identify all relevant information to define the CPM programme. As a construction project usually involves a number of activities, the validation of an as-planned programme requires a lot of effort. However, the availability of a reliable as-planned programme is prerequisite for the integrity of any conclusion. The following discussion assumes that a validated as-planned programme is available.

Let $A^{A P}$ denote the set of activities and $d_{i}^{A P}$ denote the duration of activity $i$ at the asplanned state. $\boldsymbol{P}^{A P}$ is the activity relationships. Then let $\Gamma^{A P}$ be the as-planned programme

$$
\Gamma^{A P}=\left(A^{A P},\left(d_{i}^{A P}\right)_{i \in A^{A P}}, \boldsymbol{P}^{A P}\right) .
$$

Given the as-planned programme, the as-planned project period $\tau^{A P}$ is uniquely calculated.

\subsection{As-built Programme}

The as-build programme should reflect how a contractor actually performed its work. Ideally, an as-built programme should be prepared and maintained during the course of the work. Otherwise, it cannot be prepared retrospectively unless reasonably detailed programme is not available. 
Let $A^{A B}$ denote the set of activities and $d_{i}^{A B}$ and in an as-planned programme. $\boldsymbol{P}^{A B}$ represents activity relationships of the as-built programme. Then let $\Gamma^{A B}$ be the as-built programme.

$$
\Gamma^{A B}=\left(A^{A B},\left(d_{i}^{A B}\right)_{i \in A^{A B}}, \boldsymbol{P}^{A B}\right) .
$$

Given the as-built programme, the necessary time $\tau^{A B}$ is uniquely calculated, which must coincides with the actual project period. If the actual project period and the necessary time calculated based on the as-built programme are inconsistent, it means that the as-built programme fails to reflect the actual performance.

In addition, a critical path of the as-built programme is called as 'as-built critical path'. It would be extremely rare for the logic in any large scale complex project to be exactly the same as in the as-built state as it was in the as-planned state [1].

\subsection{Identification of Delay Events}

Any delay analysis method regards the delay to completion is caused by delay event(s). A delay event is any types of event which causes delay to completion. Hence delay to completion is caused by the existence of delay events. 'Who is liable for a delay event' is a crucial question to determine the responsibility of delay. Therefore, identifying delay events is a substantial process of delay analysis.

Identification of delay events is one of the most difficult and time consuming and yet important aspects of delay analysis. Delay takes only three forms: 1) delay to commencement, 2) extended duration and 3) suspension during performance. There are two primary approaches to identify delays: effect-based approach and causebased approach [1].

The effect-based approach investigates the as-built programme to identify the deviations from the as-planned programme which are the effects of delay events. This approach relies on a strong factual matrix and chronology of the delay events which causes. The effect-based approach identifies the effects first and then investigates the causes of those effects. The cause-based approach sets a set of issues, events and potential delay events first. And then it attempts to measure the effects of those causes based on a baseline programme. The cause-based approach must be clear cut events. The cause-based approach requires a reliable as-planned programme as it must verify that identified events were not counted at the asplanned status.

Once the list of delay events is identified, the liable party for each delay event is identified. A contract document shall clearly stipulate the liable party for risk events. If delay is caused by a delay event that the Employer is liable for, the Contractor has the entitlement for extension of time. On the contrary, delay is caused by a delay event that the Contractor is liable for, the Contractor shall pay 'liquidated damage'.

Causes of delays, i.e., delay events, are typically classified into either of the Contractor's risk event or the Employer's risk event. The Contractor is entitled to have an extension of time caused by the Employer's risk events. A contract document shall clearly specify the liable party for various kinds of risk events likely to occur in construction projects. In delay analysis practice, any delay events are categorised into the following groups by the liable party and entitlement to the monetary compensation: excusable non-compensable, excusable compensable, non-excusable [5][6]. This liability for delays on construction projects is often disputed and can itself become a subject of protracted litigation [7], but these issues are beyond the scope of our study. It is assumed, that responsibility for any particular delay event is known or can be identified.

Excusable delay is a delay event for which the Contractor has the entitlement for extension of time. Excusable delay is caused by the Employer's risk event in principle. 
Non-excusable delay is a delay event for which the Contractor does not has the entitlement for extension of time. Non-excusable delay is caused by the Contractor's risk event in principle.

Another issue of delay damage is the time-related costs. Compensable delay is a period of time during which a critical delay event is experienced which is the Employer's risk event and expressly identified as being recoverable under the contract terms. Noncompensable delay is a period of time during which a critical delay event is experienced which is the Contractor's risk event and not expressly identified as being recoverable under the contract term.

This study focuses on determining the entitlement of extension of time and determining the compensation of time-related costs is out of our scope.

\section{Formalisation of Delay Analysis Methods}

\subsection{Additive Approach of Delay Analysis Methods}

The impact of delay event on the delay to completion is assessed on the basis of the 'what-if' principle. There are two ways to adopt the 'what-if' principle: 1) what would have happened if the delay event arose (additive approach) and 2) what would have happened if the delay event did not arise (subtractive approach). CPM programming is used as a tool to simulate this 'what-if' scenario.

The additive approach is to answer the question: 'what is the length of prolonged duration of project time due to the occurrence of delay event(s)?' The prerequisite of the additive approach is the availability of a reliable as-planned programme. If the as-planned programme does not reflect a reasonable scenario of the case without delay events, it is not an appropriate baseline programme to assess the effect of delay events.

The impact of delay events is assessed by revising the as-planned programme. The delay events are interpreted as newly created activities, called as fragnet, to be added to the as-planned programme or as the extension of existing activity durations.

The impact of delay events is assessed by the difference in the completion time between the two CPM programmes, i.e., pre-impacted programme and impacted programme. A pre-impacted programme is called as a baseline programme. A baseline programme is not necessary an as-planned programme. It could be a delayimpacted programme. Depending on the choice of the baseline programme, there is a variety of delay analysis methods.

Any delay event must bring the deviation from the original CPM programme. Impacts of delay events can take any forms including [1],

- delaying the start of an existing, or inserted, activity with a constraint start date;

- delaying the start of an existing, or inserted, activity with a constraint start date ;

- increasing the duration of an existing activity;

- increasing or decreasing the hours available during a working week;

- inserting a new fragnet, logically linked to existing activities in the programme; or

- adding or deleting existing logical links to reflect the impact of changed conditions and dependencies.

Formally, let $\Omega=\left\{\Omega_{1}, \cdots, \Omega_{k}, \cdots, \Omega_{K}\right\}$ denote the set of delay events of the project. Let $\Omega^{C}(\subset \Omega)$ denote the set of Contractor's risk events and $\Omega^{E}(\subset \Omega)$ the set of 
Employer's risk events. Employer's delay events and Contractor's delay events are exclusive, i.e., $\Omega^{C} \cap \Omega^{E}=\emptyset$ and $\Omega^{C} \cup \Omega^{E}=\Omega$.

In the additive approach, any impacts of delay events are interpreted as additional tasks in the CPM schedule. Formally, consider an arbitral subset of $\Omega$ denoted by $S$. Let $A^{I A P(S)}$ denote the set of activities and $d_{i}^{I A P(S)}$ and in an as-planned programme. $\boldsymbol{P}^{I A P(S)}$ is the activity relationships. Then let $\Gamma^{I A P(S)}$ be the impacted as-planned programme with the additional activities of the set of delay events $S$,

$$
\Gamma^{I A P(S)}=\left(A^{I A P(S)},\left(d_{i}^{I A P(S)}\right)_{i \in A^{I A P(S)}}, \boldsymbol{P}^{I A P(S)}\right) .
$$

Given the delay-impacted programme, the necessary time $\tau^{I A P(S)}$ is uniquely calculated, which implies the theoretical necessary time for completion given the occurrence of delay events in the subset $S$.

Consider the subset of delay events $S$ and $T$, where $T \subset S \subseteq \Omega$. Let $E(S ; T)$ be defined as

$$
E^{I A P}(S ; T)=\tau^{I A P(S)}-\tau^{I A P(T)} .
$$

$E^{I A P}(S ; T)$ denotes the additional duration of project time if delay events of $S \backslash T$ are impacted additionally to the delay-impacted programme $\Gamma^{I A P(T)}$. Here $S \backslash T=S \cup$ $\bar{T}$ and $\bar{T}$ is the complement set of $T$.

The methods which use the CPM programme with the additional impacts of delay events based on the as-planned programme is called 'impacted as-planned' [2]. It is referred as the 'Modelled/Additive/Single Base (MIP3.6)' in the AACEI RP-FSA in the US [3]. This methodology is very commonly used in practice in virtue of its simplicity. According to the SCL Protocol, the impacted as-planned is based only on the effect of the Employer's risk events on the planned programme of work. However, it is possible to consider the occurrence of the Contractor's risk events as well, which would change the analytical result from that without considering them. Then, the impacted as-planned methods are classified into two types depending on if the Contractor's risk event is considered or not.

\subsubsection{Non-integrated Impacted as-planned}

Non-integrated impacted as-planned is the impacted as-planned methods only considering the Employer's risk events. Consider the impacted as-planned programme with all delay events in $\Omega^{E}$.

$$
\Gamma^{I A P\left(\Omega^{E}\right)}=\left(A^{I A P\left(\Omega^{E}\right)},\left(d_{i}^{I A P\left(\Omega^{E}\right)}\right)_{i \in A^{I A P\left(\Omega^{E}\right)}}, \boldsymbol{P}^{I A P\left(\Omega^{E}\right)}\right) .
$$

The impacted as-planned deems the entitlement for the contractor on the extension of time as the effect of employer's risk events with the baseline programme to be the as-planned programme. Then entitlement of extension of time for the Contractor is defined as follows:

$$
E O T=E^{I A P}\left(\Omega^{E} ; \varnothing\right)=\tau^{I A P\left(\Omega^{E}\right)}-\tau^{A P} .
$$

\subsubsection{Combined Chronological Impacted As-Planned}

Contrary to non-integrated impacted as-planned which considers only the Employer's risk event, the 'combined chronological impacted as-planned' considers both of the Employer's risk events as well as the Contractor's risk events. It uses the impacted as-planned programmes adding the both of Employer's risk events and the Contractor's risk events in chronological order and the project completion date is reanalysed until all delay events in $\Omega$ have been impacted. The time of the occurrence of a delay event is effectively identified to determine the chronological order of the occurrence of delay events. 
The combined chronological impacted as-planned considers both of employer's risk events as well as contractor's risk events. Firstly, the analyst identifies the chronological order of delay events by assessing when each delay event emerged or recognised. Here the label of delay event in $\Omega=\left\{\Omega_{1}, \cdots, \Omega_{k}, \cdots, \Omega_{K}\right\}$ is identified according to the chronological order, i.e., $\Omega_{1}$ is the delay event which emerged earliest among all delay events. The first step is the assessment of delay impact of the delay event $\Omega_{1}$ with the baseline programme to be the as-planned programme. The effect of delay event $\Omega_{1}$ is

$$
E^{I A P}\left(\left\{\Omega_{1}\right\} ; \varnothing\right)=\tau^{I A P\left(\left\{\Omega_{1}\right\}\right)}-\tau^{A P} .
$$

The effect of delay event is assessed with the baseline programme as the delayimpacted programme $\Gamma^{I A P\left(\left\{\Omega_{1}\right\}\right)}$.

$$
E^{I A P}\left(\left\{\Omega_{1}, \Omega_{2}\right\} ;\left\{\Omega_{1}\right\}\right)=\tau^{I A P\left(\left\{\Omega_{1}, \Omega_{2}\right\}\right)}-\tau^{\operatorname{IAP}\left(\left\{\Omega_{1}\right\}\right)} .
$$

For the simplicity of notation, we denote $D^{I A P(k)}=E^{I A P}\left(\left\{\Omega_{1}, \cdots, \Omega_{k}\right\} ;\left\{\Omega_{1}, \cdots, \Omega_{k-1}\right\}\right)$. Combined chronological impacted asplanned defines the entitlement of extension of time by the accumulated effects of the Employer's risk events by iteratively calculating the effect of each delay event until all the delay events $\Omega$ are impacted.

$$
E O T=\sum_{k: \Omega_{k} \in \Omega^{E}} D^{I A P(k)}
$$

\subsubsection{Time Impact Analysis}

Time impact analysis is similar to impacted as-planned in the sense that both approach is additive. But time impact analysis is different from the impacted as-planned in that it updates the baseline programme during the course of project, contemporaneously reflecting the as-built status. The programme is updated every time the occurrence of a delay event recognised.

Let $T_{k}$ denote the commencement time when the occurrence of delay event $\Omega_{k}$ is recognised. The work of delay analysis is implemented at $T_{k}$. The first task at $T_{k}$ is preparation of contemporaneous programme, reflecting the as-built status until $T_{k}$ and asplanned schedule, i.e., the Contractor's intention, from $T_{k}$ to until completion. Let $\Gamma^{C P\left(T_{k}\right)}$ denote the contemporaneous programme at $T_{k}$. Hence, $\tau^{C P\left(T_{k}\right)}$, the completion time calculated based on the programme $\Gamma^{C P\left(T_{k}\right)}$ must reflect the actually spent time until $T_{k}$.

The second task at $T_{k}$ is preparation of impacted contemporaneous program adding the impacted of delay event $\Omega_{k}$. Let $\Gamma^{I C P\left(T_{k}\right)}$ denote the impacted contemporaneous programme at $T_{k}$, which is the contemporaneous programme $\Gamma^{C P\left(T_{k}\right)}$ with the estimated impact of delay event $\Omega_{k}$. It is noteworthy that the impact of delay event $\Omega_{k}$ is just an estimate at $T_{k}$ as $T_{k}$ is the time of the occurrence of delay event $\Omega_{k}$. Therefore, the duration of delay event is just an estimate, not the determined variable. Therefore, $\tau^{I C P\left(T_{k}\right)}$, the completion time calculated based on the programme $\Gamma^{I C P\left(T_{k}\right)}$, is just an expectation, but still it is the benchmark for updating the agreed contract due.

Time impact analysis assesses the effect of delay event using the contemporaneous programme $\Gamma^{C P\left(T_{k}\right)}$ as a baseline program. Then the effect of delay event $\Omega_{k}$ is assessed as follows:

$$
E^{T I A}\left(\Omega_{k}\right)=\tau^{I C P\left(T_{k}\right)}-\tau^{C P\left(T_{k}\right)} .
$$

The entitlement of extension of time is the accumulated effect of the Employer's risk event. For the simplicity of notation, we denote $D^{T I A(k)}=E^{T I A}\left(\left\{\Omega_{k}\right\}\right)$. The 
effect of each delay event is assessed iteratively in the same manner until all the delay events $\Omega$ are impacted.

$$
E O T=\sum_{k: \Omega_{k} \in \Omega^{E}} D^{T I A(k)}
$$

Time impact analysis is referred as 'modelled/additive/multiple base' by ACCEI [3]. Although both of impacted as-planned and time impact analysis is additive and modelled approach, the critical difference between them is being single or multiple of baseline programme. Impacted as-planned uses only as-planned programme as the baseline, but time impact analysis requires updates the baseline programme reflecting a temporary as-built status every time a delay event is recognised.

\subsection{Subtractive Approach of Delay Analysis Methods}

A subtractive approach of delay analysis methods relies on an as-built programme. The representative method of subtractive approach is 'collapsed as-built' method. Differently from the additive approach, the effect of delay event is assessed by subtracting delay events from the as-built programme.

Formally, consider an arbitral subset of $\Omega$ denoted by $S$. Let $A^{C A B(-S)}$ denote the set of activities, $d_{i}^{C A B(-S)}$ denote the duration of activity $i \in A^{C A B(-S)}$ and $\boldsymbol{P}^{C A B(-S)}$ denote the activity relationships when the delay events in subset $S$ are subtracted from the asbuilt programme. Then let $\Gamma^{C A B(-S)}$ be the collapsed as-built programme defined as

$$
\Gamma^{C A B(-S)}=\left(A^{C A B(-S)},\left(d_{i}^{C A B(-S)}\right)_{i \in A^{C A B(-S)}}, P^{C A B(-S)}\right) .
$$

In a similar way as the impacted as-planned, there are two types of defining the entitlement depending on the inclusion of the Contractor's risk events into consideration. The non-integrated collapsed as-built defines the entitlement for the Contractor on the extension of time as the effect of the Employer's risk events with the baseline programme to be the as-built programme, that is,

$$
E O T=E^{C A B}\left(\Omega^{E} ; \varnothing\right)=\tau^{A B}-\tau^{C A B\left(-\Omega^{E}\right)} .
$$

The combined chronological approach subtracts both of the Employer's and the Contractor's risk event according to the inverse chronological order.

The collapsed process starts from subtracting delay event $\Omega_{K}$ from the as-built programme. The effect of delay event $\Omega_{K}$ is defined as

$$
E^{C A B}\left(\left\{\Omega_{K}\right\} ; \varnothing\right)=\tau^{A B}-\tau^{C A B\left(-\left\{\Omega_{K}\right\}\right)} .
$$

Then the effect of delay event $\Omega_{k}$ is assessed by comparing the completion time between the collapsed as-built programmes $\Gamma^{C A B\left(-\left\{\Omega_{K}, \Omega_{K-1}, \cdots, \Omega_{k+1}\right\}\right)}$ and $\Gamma^{C A B\left(-\left\{\Omega_{K}, \Omega_{K-1}, \cdots, \Omega_{k}\right\}\right)}$.

$$
\begin{aligned}
& E^{C A B}\left(\left\{\Omega_{K}, \Omega_{K-1}, \cdots, \Omega_{k}\right\} ;\left\{\Omega_{K}, \Omega_{K-1}, \cdots, \Omega_{k+1}\right\}\right) \\
= & \tau^{C A B\left(-\left\{\Omega_{K}, \Omega_{K-1}, \cdots, \Omega_{k+1}\right\}\right)}-\tau^{C A B\left(-\left\{\Omega_{K}, \Omega_{K-1}, \cdots, \Omega_{k}\right\}\right)}
\end{aligned}
$$

For the simplicity of notation, we denote

$$
D^{C A B(k)}=E^{C A B}\left(\left\{\Omega_{K}, \Omega_{K-1}, \cdots, \Omega_{k}\right\} ;\left\{\Omega_{K}, \Omega_{K-1}, \cdots, \Omega_{k+1}\right\}\right)
$$

Then the entitlement for the extension of time is defined as the accumulated effect of the Employer's risk events by iteratively calculating the effect of each delay event until all the delay events $\Omega$ are subtracted.

$$
E O T=\sum_{k: \Omega_{k} \in \Omega^{E}} D^{C A B(k)} .
$$




\section{Conclusion}

A variety of delay analysis methods have been innovated in practice. Although academic papers and guides published by authoritative societies introduce the protocol of delay analysis methods, those are described in the natural language. However, the description in the natural language is likely to create the ambiguity in actual implementation and hence the room for the manipulation of analytical result. Being tempted by such a motive, this paper formalised typical delay analysis methods including impacted as-planned, time impact analysis and collapsed as-built commonly used in practice. The advantage of formal description is not only to avoid the ambiguity of description but also to identify critical mechanisms which differentiate delay analysis methods. Those methods apply 'what-if' principle, comparing the completion date between the case with delay event(s) and the one without. The methods are differentiated by 1) the consideration of Contractor's risk events and 2) the choice of baseline programme, i.e., as-planned programme, as-built programme or contemporary updated programme. In conclusion, this study proposed a formal description of delay analysis methods that would be applicable to any types of them so that delay analysts can avoid any confusion caused by the 'name' approach.

\section{References}

[1] T. Farrow, "Delay analysis - Methodology or Mythology", Society of Construction Law, (2001).

[2] P. J. Keane and A. F. Caletka, "Delay Analysis in Construction Contracts", Wiley-Blackwell, (2008).

[3] Society of Construction Law, Delay and Disruption Protocol, (2002).

[4] The Association for the Advancement of Cost Engineering International, Recommended Practice No. 29R-03 Forensic Schedule Analysis, (2007).

[5] Project Management Institute: The Guide to the Project Management Body of Knowledge, 4th Edition, (2008).

[6] R. A. Rubin, S. D. Guy, A. C. Maevis and V. Fairweather, "Construction Claims, Analysis, Presentation and Defence", Van Nostrand Reinhold, (1983).

[7] B. B. Bramble and M. T. Callahan, "Construction Delay Claims", Wiley Law Publications, (2009).

[8] J, K. Yates and A. Epstein, "Avoiding and minimizing construction delay claim disputes in relational contracting", Journal of Professional Issues in Engineering Education and Practice, vol. 132, no. 2, (2006), pp. 168-179.

[9] M. R. Finke, "Window analysis of compensable delays", Journal of Construction Engineering and Management, vol. 125, no. 2, (1999), pp. 96-100.

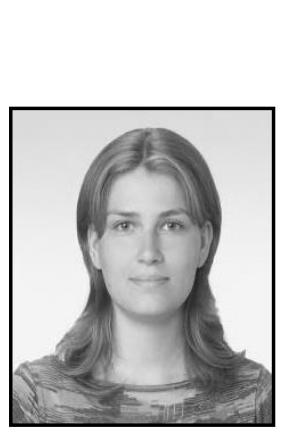

\section{Authors}

Alena Vasilyeva-Lyulina, Dr. Eng, Advisor at Project Advisors International LLC. She is a recent graduate from Kyoto University (Japan), where she did research on delay analysis and was awarded a doctorate degree. Originally from Russia, she graduated from Plekhanov Russian Academy of Economics and earned the degree of planning engineer which is evaluated as an equivalent of BSc. degree in business administration with an emphasis on the construction industry and a MSc. degree in construction management. Her research interests include Project Management, Project Scheduling and Planning, Project Delay Analysis and Dispute Resolution.

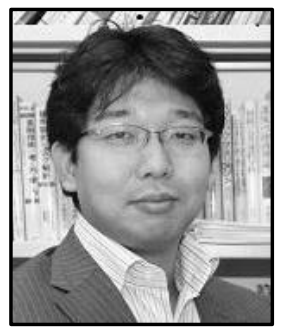

Masamitsu Onishi, BSc., MSc., Dr Eng., is Assistant Professor at the Department of Urban Management, Graduate School of Engineering, Kyoto University, Japan. His doctoral degree was awarded by Kyoto University. He has published 40 academic papers which concerns dispute resolution in construction projects, public 
private partnership, project financing. Dr. Onishi is a board member of Research Committee of Private Finance Initiative in Japan Society of Civil Engineering (JSCE). He is a recipient of JSCE young researcher award 2006 and best paper award 2008.

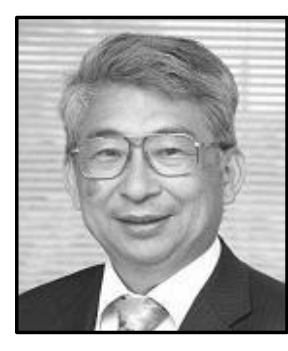

Kiyoshi Kobayashi, BSc., MSc., Dr Eng., Kyoto University, is a Professor of infrastructure economics at Kyoto University. He serves on the editorial boards of the American Society of Civil Engineers, the Annals of Regional Science, the Journal of Applied Regional Science and the Japan Journal of Civil Engineering. He was also the Editor in chief for the Journal of Japan Society of Civil Engineers and Vice Editor in chief, Journal of Infrastructure Systems. He is the author and co-editor of 53 books and more than 370 technical papers. 